Life Sciences and Medicine

Special Topic: COVID-19: Virus, Immunity and Vaccines

\title{
Towards robust immune responses after heterologous COVID-19 vaccination and its application perspectives
}

\author{
Yong Lin ${ }^{*} \&$ Ailong Huang* \\ Key Laboratory of Molecular Biology of Infectious Diseases (Ministry of Education of China), Chongqing Medical University, Chongqing \\ 400016, China \\ *Corresponding authors (emails: linyong@cqmu.edu.cn (Yong Lin); ahuang@cqmu.edu.cn (Ailong Huang))
}

Received 8 October 2021; Revised 22 December 2021; Accepted 5 January 2022; Published online 25 March 2022

The coronavirus disease 2019 (COVID-19) pandemic, caused by severe acute respiratory syndrome coronavirus 2 (SARS-CoV-2) [1], has seriously impacted the global health and economy. Effective vaccination, with homologous or heterologous prime-boost strategies, is the key to controlling the ongoing COVID-19 pandemic [2]. Homologous vaccinations, which are the administration of the same type of COVID-19 vaccine, have been shown to be highly efficient in inducing robust immune responses [3-7]. Due to safety concerns regarding the rare cases of vaccine-induced immune thrombotic thrombocytopenia after the first dose of the ChAdOx1 nCov-19 (AZD1222) vaccine [8], heterologous booster vaccination was assessed and approved in some European countries. There are limited data on the immune responses induced by heterologous vaccination regimens. We summarize the latest data on the immunogenicity, reactogenicity, and neutralizing activity of heterologous COVID-19 vaccinations against variants of concern (VoCs); we further discuss the distinct advantages of their application and critical factors that may influence their efficiency.

Currently, different countries have approved multiple types of COVID-19 vaccines, including mRNAbased vaccines (BNT162b2/BNT and mRNA-1273), adenovirus-vectored vaccines (ChAdOx1 nCov-19/ ChAd, Janssen Ad26.COV2.S, and Gam-COVID-Vac), inactivated virus vaccines (Sinovac-CoronaVac and Sinopharm-BBIBP-CorV), and protein subunit vaccines (ZF2001) $[2,9,10]$. Some of these vaccines have shown high efficacy and safety profiles. The large-scale use of some COVID-19 vaccines has led to the emergence of critical challenges, such as vaccine supply shortage and safety concerns. Therefore, in some countries, the use of homologous vaccinations has been restricted, and heterologous booster vaccinations with vaccines, such as BNT and ZF2001, have been considered. In this alternative vaccination strategy, mRNA or protein subunit vaccines may be used as a booster against COVID-19 to replace the homologous booster immunization with adenovirus-vectored, inactivated virus, or other vaccines.

\section{Humoral immune responses after a heterologous vaccination}

Although a heterologous vaccination is a promising alternative strategy, its immunogenicity and re-

(C) The Author(s) 2022. Published by China Science Publishing \& Media Ltd. and EDP Sciences. This is an Open Access article distributed under the terms of the Creative Commons Attribution License (https://creativecommons.org/licenses/by/4.0), which permits unrestricted use, distribution, and reproduction in any medium, provided the original work is properly cited. 
actogenicity remain largely unknown. To date, there are limited studies comparing immune responses to homologous and heterologous vaccination schedules in humans (Table 1). In February 2021, Logunov et al. [3] reported the interim clinical efficacy results of a heterologous prime-boost vaccination regimen using the rAd26- and rAd5-vectored COVID-19 vaccine Gam-COVID-Vac separately in a randomized, double-blind, placebo-controlled multicentre study. The study found that this rAd26/rAd5 vector-based heterologous vaccination induced robust humoral and cellular immune responses with good safety profiles and had a 91.6\% protective efficacy against COVID-19. Homologous immunizations with the ChAd or BNT vaccines have been evaluated in American and European countries. Two observational studies of heterologous primeboost vaccination regimens, incorporating ChAd and BNT vaccines, have been conducted in healthy adult individuals in Germany [4,5]. In late July 2021, Tenbusch et al. [5] reported the vaccine-induced antibody responses in healthcare workers or volunteers who received homologous or heterologous vaccinations using ChAd and BNT as a prime and/or boost vaccine in Germany. Interestingly, the heterologous ChAd/BNT vaccination at a 10-week interval induced a significantly higher surrogate neutralization activity than homologous ChAd/ChAd (at a 10-week interval) or homologous BNT/BNT (at a 3-week interval) vaccination. In September 2021, Schmidt et al. [4] reported that a heterologous ChAd/BNT vaccination (10-week interval) induced higher T-cell responses than a homologous BNT/BNT vaccination (3-week interval); nevertheless, the two vaccination strategies yielded similar levels of anti-spike (S) IgG and neutralizing antibodies, consistent with the findings of Tenbusch et al. [5]. Moreover, two critical clinical trials, with 1,506 participants from Spain and the UK, were conducted to study the immunogenicity and reactogenicity of heterologous COVID-19 vaccinations [6,7]. Borobia et al. [6] conducted the CombiVacS study, a multicentre, open-label, randomized, controlled phase 2 trial in Spain. The study demonstrated significant increases in SARS-CoV-2 anti-S IgG and neutralizing antibody levels and T-cell responses in the ChAdprimed, BNT-boosted participants compared with those in participants not administered a BNT booster, which indicated that humoral immune responses increased following a BNT booster vaccination. However, this study lacked the data regarding the homologous ChAd/ChAd vaccination comparator. Liu et al. [7] also observed in their single-blind, randomized, noninferiority trial that heterologous ChAd/BNT and homologous BNT/BNT vaccinations induced higher immunogenicity than a homologous ChAd/ChAd vaccination did. The participants who were vaccinated with ChAd/BNT or BNT/BNT had similar levels of anti-S IgG and neutralizing antibodies, while T-cell responses were higher in the ChAd-primed, BNT-boosted participants. Although both heterologous vaccinations (ChAd/BNT and BNT/ChAd) led to higher levels of anti-S IgG than that induced by the homologous $\mathrm{ChAd} / \mathrm{ChAd}$ vaccination comparator, the BNT/ChAd regimen did not meet the noninferiority criteria [8]. Collectively, accumulating evidence suggests that heterologous COVID19 vaccinations with ChAd and BNT boost the vaccine's effectiveness by triggering stronger or broader immune responses.

Recently, the rapid worldwide spread of the B.1.617.2 (Delta) variant has partially nullified the protection effectiveness afforded by COVID-19 vaccines, suggesting that booster vaccination would be beneficial for combating emerging VoCs. Keskin et al. designed a small clinical trial to investigate the differences of in antibody titres to $\mathrm{S}$ and nucleocapsid $(\mathrm{N})$ proteins in 45 healthcare workers who already received two doses of an inactivated virus vaccine (2IVV) and were administered a third dose of the CoronaVac (3IVV) or BNT $(2 \mathrm{IVV}+\mathrm{BNT})$ vaccines in Turkey [11]. They found that the levels of anti-S and anti-N IgG antibodies in the $2 \mathrm{IVV}+\mathrm{BNT}$ group were significantly higher than those in the 3IVV group. Notably, two recent clinical 
Natl. Sci. Open, 2022, Vol.1, 20220008

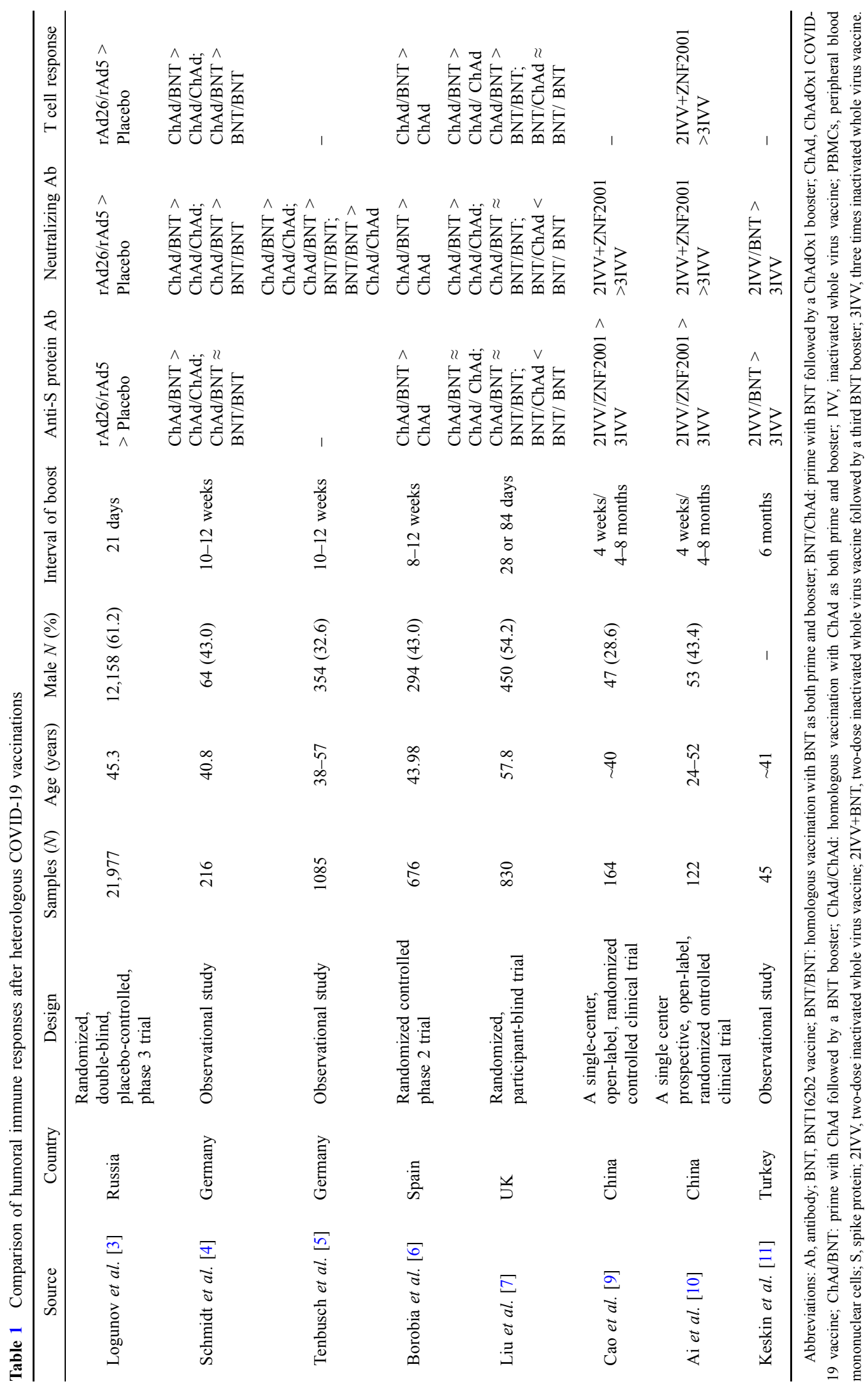


studies separately, conducted by Cao et al. [9] and Ai et al. [10], evaluated the safety and immunogenicity of a heterologous third booster dose with the recombinant protein subunit vaccine ZF2001 primed with two doses of IVVs (CoronaVac or BBIBP-CorV) in China. Both studies showed that the third booster dose of ZF2001 was well tolerated and could induce a significantly high humoral immunogenicity in adult recipients. Therefore, these studies indicate that a heterologous third booster dose induces a much stronger B-cell recall response than its homologous counterpart.

To date, there are limited data regarding the neutralization capacities of heterologous vaccination regimens against different emerging VoCs [12], including B.1.1.7 (Alpha), B.1.351 (Beta), P.1 (Gamma, formerly B.1.1.28.1), and B.1.617.2 (Delta) variants. Barros-Martins et al. [13] reported that the heterologous ChAd/ BNT vaccination elicited significantly higher neutralizing antibody titres against the B.1.1.7, B.1.351, and P.1 variants than homologous $\mathrm{ChAd} / \mathrm{ChAd}$ vaccination. Interestingly, their subsequent study further found that the homologous BNT/BNT vaccination more efficiently induced neutralizing antibodies against the B.1.617.2 variant than the heterologous ChAd/BNT vaccination. However, the heterologous ChAd/BNT vaccination also supported strong inhibition of the B.1.617.2 variant by inducing robust neutralizing antibodies. Recently, two independent clinical studies reported that a heterologous third booster dose with ZF2001 was more efficient than a homologous third booster dose with IVVs in terms of inducing humoral immunity against different SARS-CoV-2 variants, including the Delta strain [9,10]. Heterologous primeboost COVID-19 vaccination as well as additional heterologous vaccine doses might be a suitable strategy to combat emerging VoCs. Therefore, there is an urgent need for more detailed studies to evaluate and compare the immunogenicity of heterologous and homologous COVID-19 vaccinations against emerging VoCs.

\section{Distinct advantages of heterologous vaccinations}

Given the ongoing COVID-19 pandemic, heterologous COVID-19 vaccination may have distinct advantages. First, mRNA vaccines may serve as an alternative heterologous booster for the ChAd vaccine to overcome their safety concerns. Second, heterologous vaccinations may boost more durable and robust humoral and cellular immune responses than those induced by already authorized homologous vaccinations and greatly contribute to effective control of emerging or future VoCs $[4,6,10]$. Finally, as a feasible and reasonable vaccination strategy, heterologous vaccinations also have the potential to compensate for vaccine shortfalls and accelerate the worldwide vaccine rollout, especially in low- and middle-income countries.

\section{Factors influencing the efficiency of heterologous vaccinations}

Some of the critical factors that may influence the high efficiency of heterologous COVID-19 vaccinations are as follows: 1) the vaccine types used in the heterologous prime-boost schedules [9-11]; 2) the order of candidate vaccines in the prime-boost process of a heterologous vaccination regimen [7]; and 3) the differences in time interval between doses of the vaccines in heterologous vaccinations [14].

Overall, accumulating evidence supports the fact that certain heterologous COVID-19 vaccination regimens have good safety profiles and represent a feasible and promising vaccination strategy to boost durable 
and robust humoral and cellular immune responses and effectively control the emerging VoCs $[4,6,10]$. Notably, a feasible heterologous vaccination is a powerful weapon against the ongoing COVID-19 pandemic. Further studies are needed to confirm the clinical effectiveness of heterologous vaccinations against emerging VoCs and optimize the combinations, orders, doses, and intervals of heterologous prime-boost vaccination schedules.

\section{Funding}

This work was supported by the COVID-19 Emergency Project from Chongqing Medical University (CQMUNCP0207).

\section{Author contributions}

AH conceived this article; YL wrote the original draft and prepared the table; YL and AH edited and reviewed the manuscript.

\section{Conflict of interest}

The authors declare that they have no conflict of interest.

\section{References}

1 Zhou P, Yang XL, Wang XG, et al. A pneumonia outbreak associated with a new coronavirus of probable bat origin. Nature 2020; 579: 270-273.

2 Chiu NC, Chi H, Tu YK, et al. To mix or not to mix? A rapid systematic review of heterologous prime-boost covid-19 vaccination. Expert Rev Vaccines 2021; 20: 1211-1220.

3 Logunov DY, Dolzhikova IV, Shcheblyakov DV, et al. Safety and efficacy of an rAd26 and rAd5 vector-based heterologous prime-boost COVID-19 vaccine: an interim analysis of a randomised controlled phase 3 trial in Russia. Lancet 2021; 397: 671-681.

4 Schmidt T, Klemis V, Schub D, et al. Immunogenicity and reactogenicity of heterologous ChAdOx1 nCoV-19/mRNA vaccination. Nat Med 2021; 27: 1530-1535.

5 Tenbusch M, Schumacher S, Vogel E, et al. Heterologous prime-boost vaccination with ChAdOx1 nCoV-19 and BNT162b2. Lancet Infect Dis 2021; 21: 1212-1213.

6 Borobia AM, Carcas AJ, Pérez-Olmeda M, et al. Immunogenicity and reactogenicity of BNT162b2 booster in ChAdOx1-S-primed participants (CombiVacS): a multicentre, open-label, randomised, controlled, phase 2 trial. Lancet 2021; 398: 121-130.

7 Liu X, Shaw RH, Stuart ASV, et al. Safety and immunogenicity of heterologous versus homologous prime-boost schedules with an adenoviral vectored and mRNA COVID-19 vaccine (Com-COV): a single-blind, randomised, noninferiority trial. Lancet 2021; 398: 856-869.

8 Greinacher A, Thiele T, Warkentin TE, et al. Thrombotic thrombocytopenia after ChAdOx1 nCov-19 vaccination. $N$ Engl J Med 2021; 384: 2092-2101.

9 Cao Y, Hao X, Wang X, et al. Humoral immunogenicity and reactogenicity of CoronaVac or ZF2001 booster after two doses of inactivated vaccine. Cell Res 2022; 32: 107-109.

10 Ai J, Zhang H, Zhang Q, et al. Recombinant protein subunit vaccine booster following two-dose inactivated vaccines dramatically enhanced anti-RBD responses and neutralizing titers against SARS-CoV-2 and variants of concern. Cell Res 2022; 32: 103-106.

11 Keskin AU, Bolukcu S, Ciragil P, et al. SARS-CoV-2 specific antibody responses after third CoronaVac or BNT162b2 vaccine following two-dose CoronaVac vaccine regimen. $J$ Med Virol 2022; 94: 39-41.

12 Abdool Karim SS, de Oliveira T. New SARS-CoV-2 variants - clinical, public health, and vaccine implications. $N$ Engl $J$ Med 2021; 384: 1866-1868. 
13 Barros-Martins J, Hammerschmidt SI, Cossmann A, et al. Immune responses against SARS-CoV-2 variants after heterologous and homologous ChAdOx1 nCoV-19/BNT162b2 vaccination. Nat Med 2021; 27: 1525-1529.

14 World Health Organization. COVID-19 vaccine tracker and landscape. https://wwwwhoint/publications/m/item/draftlandscape-of-covid-19-candidate-vaccines. 2021. 